\title{
Aux origines de la réglementation française actuelle en matière de mouvements de versants : la coulée du plateau d'Assy en 1970
}

4, rue du Jouffrey 38180 Seyssins antoine.pi@free.fr

\section{J. DEBELMAS}

10, chemin Carronnerie $38700 \mathrm{La}$ Tronche jacques.debelmas@orange.fr

\section{J.-L. DURVILLE}

$M E E D D M-C G E D D$

Tour Pascal B 92055 La Défense Cedex jean-louis.durville@ developpement-durable. gouv.fr
Un sanatorium du plateau d'Assy (Haute-Savoie) fut partiellement détruit par un glissement-coulée en avril 1970, causant la mort de 71 personnes, presque exclusivement des enfants. La recherche des causes de cette catastrophe et des éventuelles responsabilités mit en évidence le manque de connaissance scientifique sur les instabilités des versants naturels et la faible perception du danger chez les autorités comme chez les particuliers. L'absence d'une réglementation adaptée en matière d'urbanisme en découlait et le cadre juridique de la gestion des risques était très insuffisant.

A partir de ces constatations et pour combler les lacunes ainsi révélées, des méthodes d'évaluation et de cartographie des aléas ainsi que des réglementations spécifiques furent peu à peu établies, ce qui nous fait considérer l'année 1970 comme une date charnière, en France, pour la gestion du risque de mouvement de terrain.

Le glissement-coulée du plateau d'Assy reste un phénomène exceptionnel, considéré comme imprévisible à l'époque ; il en serait probablement de même aujourd'hui, en particulier pour ce qui est de la distance parcourue par la coulée.

Mots-clés : glissement, coulée, cartographie d'aléa, réglementation, Assy.

\section{The present French regulation about landslide hazard originates from the 1970 Plateau d'Assy landslide}

A sanatorium located on the Plateau d'Assy (Haute-Savoie) was partially destroyed in April 1970 by a flow slide, resulting in the death of 71 people, most of them being children. The flow slide was considered unforeseeable by the experts; it would not be different today particularly as far as the run-out distance is concerned. Looking for the causes of this catastrophic event and for the possible responsibilities evidenced the lack of scientific knowledge about landslides and hazard mapping, and of perception of the danger linked to the geological hazards in mountainous areas. This resulted in an absence of a well-fitted land use regulation.

In order to fill the gap underlined by this catastrophic event, landslide hazard mapping has developed and specific rules about land use were progressively established, that make us consider the year 1970 as a turning point in the French management of landslide hazards.

Key words: landslide, flow slide, hazard mapping, regulation, Assy. 
Les mouvements de terrains, d'ampleur variable, affectent depuis toujours les activités humaines. Autrefois, lorsque notre pays était beaucoup moins peuplé et les moyens d'information quasi inexistants, seuls les plus spectaculaires d'entre eux frappaient les imaginations et faisaient l'objet de quelques narrations par des spectateurs ou des chroniqueurs. Il en fut ainsi pour le célèbre écroulement de la falaise nord du mont Granier, à l'extrémité septentrionale du massif de la Chartreuse, survenu en 1248, lequel aurait causé un très grand nombre de victimes. La catastrophe est rapportée (entre autres) par un moine bénédictin anglais qui la considère comme la conséquence des pratiques d'usure et de débauche des Savoyards, habitués (d'après lui) à égorger et à piller les voyageurs et les pèlerins (Pachoud, 1983). Deux siècles plus tard, en 1442, un nouveau phénomène spectaculaire se manifesta plus au sud avec l'écroulement du Claps de Lucen-Diois qui barra le cours de la Drôme, ce qui provocua la formation de deux lacs (apparemment sans causer de victimes, mais seulement la perte de riches terres agricoles - ce qui était fort grave dans cette région rocheuse et aride et par conséquent pauvre). Le plus grand, d'une surface de 17 ha environ, subsista jusqu'en 1788. A cette date il fut asséché par les moines de la Chartreuse de Durbon (Froment, 1983).

A ces époques anciennes les hautes vallées alpines n'étaient guère peuplées et le sort de leurs populations n'intéressait pas grand monde. Les deux catastrophes précédemment citées ont eu lieu dans des montagnes de moindre altitude, plus propices à la colonisation humaine, et ont donc suscité des témoignages. On voit alors se dessiner la trame de ce que l'on appellera plus tard le risque de ( mouvement de versant $)$ : il faut des pentes importantes et des terrains propices, ainsi que des victimes potentielles.

Tout ceci était cependant plus ou moins négligé par la puissance publique jusqu'à ce que « l'aménagement de la montagne ), pour des raisons économiques, entraîne un véritable urbanisme montagnard avec le développement d'agglomérations touristiques et thermales.

Vers les années 50, on vit se développer des stations de ski de plus en plus ambitieuses, ce qui eut comme conséquence la multiplication de bâtiments en dur dans des lieux considérés quelques décennies auparavant comme inhospitaliers. Ces aménagements nécessitaient la réalisation ou l'amélioration de nombreuses voies de desserte routières qui allaient souvent se trouver à l'origine d'instabilités de versants ou bien en subir les conséquences. Les aménageurs responsables de ces premiers projets étaient établis dans des métropoles lointaines et connaissaient mal le milieu montagnard. Les premiers ennuis se manifestèrent rapidement et il fallut rechercher des responsables. Il apparut que les spécialistes des milieux naturels montagnards que sont les géologues, tout autant que les fonctionnaires chargés de la sécurité publique, manquaient de références et de repères en la matière. Ils devaient rapidement procéder à une mise à jour de leurs connaissances, ce qui revenait pour les uns (les géologues) à s'intéresser à des phénomènes qu'ils ne considéraient guère jusque-là, et pour les autres (les fonctionnaires) à constater qu'ils ne disposaient pas d'outils réglementaires et juridiques assez précis pour déterminer équitablement des responsabilités en la matière.
En 1970, deux dramatiques accidents ont lieu dans les Alpes du Nord : l'avalanche de Val-d'Isère et le glissement-coulée du plateau d'Assy, faisant respectivement 39 et 71 victimes.

$C^{\prime}$ est une situation fréquente dans le monde que des catastrophes engendrent, de la part de la puissance publique, des actions techniques et réglementaires. Citons simplement trois exemples anciens :

- entre 1774 et 1776 , des effondrements de carrières souterraines à Paris conduisent Louis XVI à créer le service de l'Inspection générale des carrières en 1777 ;

- les crues du milieu du XIX siècle dans les grands cours d'eau français ont provoqué des débats sur l'érosion en montagne et sur la nécessité de reboisement: les lois sur le reboisement en 1860 et sur la restauration des terrains en montagne (RTM) en 1882 en sont issues ;

- le séisme d'Orléansville (aujourd'hui Ech-Cheliff) en 1954 a été à l'origine des premières règles « antisismiques os françaises.

Le présent article se limite aux risques de mouvement de terrain et aux cinquante dernières années, en détaillant l'événement du plateau d'Assy et ses répercussions.

\section{La situation avant 1970}

\section{Les connaissances géologiques}

Le document de base est la carte géologique de la France dont le levé a débuté au milieu du XIX ${ }^{\mathrm{e}}$ siècle. Pour la région de Grenoble par exemple, les premières cartes (échelle 1/80 000 avec fond topographique en hachures) furent disponibles à partir de 1852 (Debelmas, 2009).

Ces cartes étaient en fait des documents d'exploration réalisés dans des conditions très difficiles, qui s'attachaient à représenter la stratigraphie et la structure des assises rocheuses. La représentation des formations et phénomènes quaternaires n'était évidemment pas la priorité. Par ailleurs, le fond topographique en hachures de la carte, dite d'État-Major, ne se prêtait pas à des levés détaillés et précis. On notera que sur la première édition de la feuille Chambéry (1901), le grand écroulement du Granier, s'il est bien mentionné en tant que tel dans la notice explicative, n'est figuré sur la carte que sous le symbole (Éboulis ». Sa délimitation est par contre excellente si l'on considère la médiocrité du fond topographique.

La figuration du relief en courbes de niveau, si elle a permis des levés beaucoup plus détaillés à l'échelle $1 / 20000$ transcrits pour l'édition à 1/50 000, n'a pas conduit immédiatement à une meilleure prise en compte du Quaternaire et notamment des mouvements de terrains. La ( grande géologie » restait la préoccupation majeure des cartographes, mais cela était aussi à mettre au compte du manque de préparation des esprits à reconnaître et interpréter les formes liées aux mouvements de terrain, l'ampleur de certains phénomènes dépassant la perception des auteurs de cartes.

C'est ainsi par exemple que, sur la feuille Chambéry publiée en 1963, ne figure pas comme tel, le vaste mou- 
vement qui affecte le versant ouest de la montagne de l'Épine autour de la localité de Verthemex. Toutes les cartes antérieures à 1960 présentent de nombreuses lacunes de ce type qu'il serait fastidieux d'énumérer ici.

Il aura fallu l'exécution de grands travaux de génie civil dans les vallées alpines, pour les aménagements hydroélectriques ou l'amélioration du réseau routier, pour révéler aux géologues l'existence de mouvements de versants de grande ampleur. Il existe même des cas d'interprétation structurale erronée, comme celui rapporté par Antoine (1988) : à Jausiers en Ubaye, le redoublement du flysch à Helminthoïdes entre le sommet de Costebelle et sa base avait été attribué à un pli (Fig. 1), alors que le pseudo-flanc inverse basal est en fait le résultat d'un énorme glissement de versant (Kerckhove, 1971).

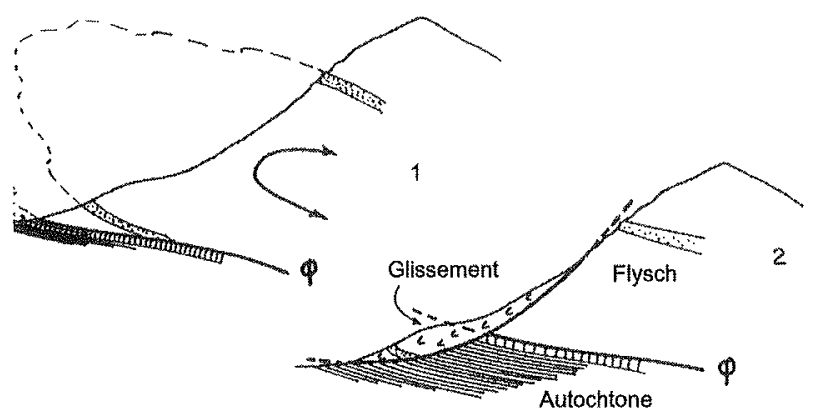

16. 1 Coupes du glissement de Jausiers l'interprétation des anciens auteurs (1) et celle de C. Kerckhove (2).

Section of the Jausiers landslide : initial (1) and correct (2) interpretation.

Mais c'est un projet de barrage sur la Haute Romanche, en contrebas de Villar-d'Arêne, étudié par M. Gignoux pendant la dernière guerre (1941 à 1943), qui apporta la preuve, pour la première fois, de la réalité de grands mouvements de versants. Un sondage réalisé en pied de la rive droite de la Romanche (Antoine et Pachoud, 1976), après avoir traversé quatre-vingts mètres de schistes liasiques, retrouva des alluvions de la Romanche, par conséquent sous des formations glissées (Fig. 2).
Mentionnons également que les innombrables glissements qui affectent la vallée de l'Arc au franchissement de la zone houillère alpine n’ont été identifiés et étudiés que récemment avec la réalisation de voies autoroutières et notamment de la route d'accès au tunnel du Fréjus.

\section{9}

\section{Les textes législatifs et réglementaires}

Un texte très ancien du Code des communes énonce que la police municipale comprend notamment « le soin de prévenir, par des précautions convenables [...], les éboulements de terre ou de rochers m. L'application qui en est faite répond le plus souvent à une situation d'urgence, où l'évacuation des habitations menacées de façon grave et imminente s'impose. En 1961, un décret précise que la constructibilité d'un terrain peut être soumise à condition : « La construction sur des terrains exposés à un risque naturel tel que inondation, érosion, affaissement, éboulement, avalanches, peut, si elle est autorisée, être subordonnée à des conditions spéciales ». En dehors du département de l'Isère, précurseur en la matière, où des cartes de risques naturels communales ont été réalisées à partir de 1967 (Besson, 2005), ce texte était très peu appliqué.

\section{2}

\section{La catastrophe du plateau d'Assy}

\section{2.}

\section{Rappel des faits ${ }^{1}$}

Le 16 avril 1970 à 0 h 15, un glissement de terrain descendant des contreforts du massif de Plate (vallée de l'Arve en Haute-Savoie), sur une dénivellation de

(1) D’après le rapport inédit de J. Debelmas : Rapport sur les conditions géologiques du glissement de terrain du plateau d'Assy, commune de Passy (Haute-Savoie), 1970. Il est frappant de constater qu'aucune étude détaillée du site n'a été entreprise suite à la catastrophe.

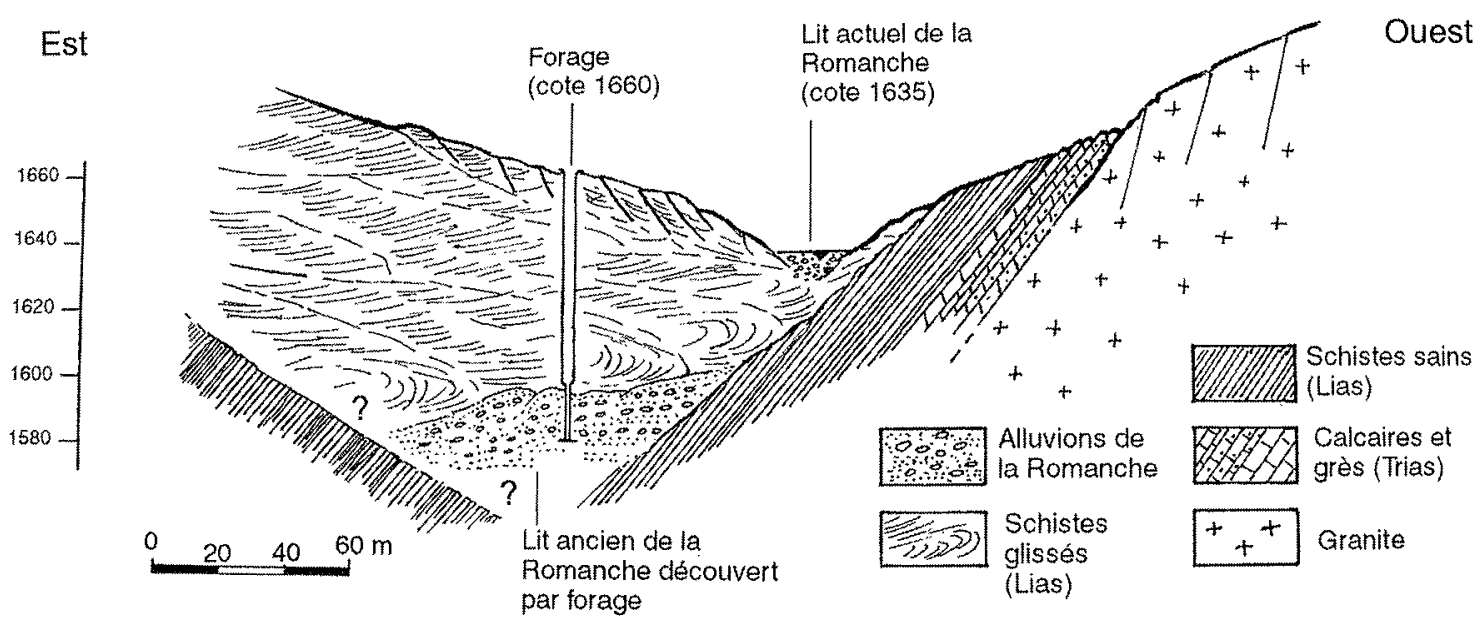

Coupe du glissement de Villar-d'Arène (d'après Besson, 2005). Section of the Villar-d'Arène landslide. 
$300 \mathrm{~m}$ pour une longueur parcourue de $500 \mathrm{~m}$, a détruit l'aile ouest du sanatorium du Roc des Fiz, au lieu-dit Praz-Coutant, causant la mort de 71 personnes, majoritairement des enfants, ce qui horrifia les populations aussi bien localement qu'à l'échelle nationale (Fig. 3).

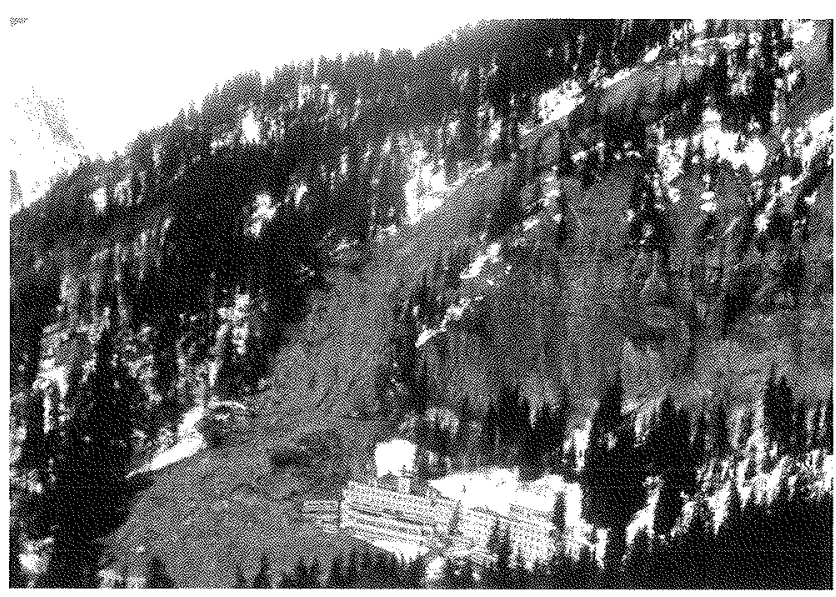

8. 16.3 Vue générale de la coulée du plateau d'Assy General view of the Plateau d'Assy flow slide.

Le préfet de Haute-Savoie diligenta une expertise assurée par l'un de nous (J.D.), à l'époque directeur du laboratoire de géologie alpine à Grenoble, en lui fixant la mission suivante

«-Quelle est la cause du glissement de terrain ? Peutil continuer de s'étendre? Était-il prévisible tant au moment de la construction du sanatorium qu'à la veille de la catastrophe?

- Y a-t-il une relation entre une coulée de neige observée le 5 avril et le glissement de terrain du 16? »

Ces deux questions cernent bien les préoccupations du représentant de l'État dans un domaine qui ne lui était pas familier. Il désirait être éclairé sur les points suivants :

- la détermination des causes (car, en principe, en droit, à toute cause identifiée doit exister un remède) ;

- était-il possible de prévoir la poursuite et l'extension de la coulée (pour décider de l'évacuation des bâtiments restants)?

- le mouvement de terrain était-il prévisible au moment de la construction d'une part, ou quelques jours avant la catastrophe d'autre part? Ici le souci du préfet est la recherche d'éventuelles responsabilités avec, sans doute, une idée sous-jacente : y a-t-il eu une lacune dans le " porté à connaissance » et donc faute de quelqu'un ou de l'administration?

Le rapport géologique de l'expert (J.D.) s'est efforcé de reconstituer avec les seuls moyens d'observation du terrain et son expérience de la montagne, le contexte géologique et le mécanisme qui a conduit à la catastrophe.

\section{3.}

\section{Les conditions géologiques}

\section{(x)}

Le glissement s'est déclenché aux alentours de la cote 1550 , en contrebas d'une falaise, sur un talus natu- rel dont l'inclinaison est de l'ordre de $35^{\circ}$. La pente du versant est exposée au sud-ouest; en dessous de la cote 1450 commence à se dessiner un vallonnement qui donne peu à peu naissance à un petit talweg, lequel sous la cote 1400 prend une orientation franchement nord-sud (descendant vers le sud).

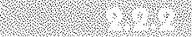 \\ Lithologie}

La coupe géologique relevée depuis le sanatorium et au-dessus montre (Figs. 4 et 5 ) :

- à la base et dominant immédiatement le sanatorium (Fig. 3), une falaise claire faite de calcaires durs et compacts appartenant au Jurassique supérieur (Tithonien). Cette falaise s'amenuise vers l'ouest au franchissement du petit talweg d'orientation nord-sud mentionné cidessus. Elle y est probablement morcelée et pratique ment recouverte de végétation ;

- au-dessus vient un premier ensemble de couches calcaréo-marneuses, épais d'environ $120 \mathrm{~m}$, de la base du Crétacé inférieur (Berriasien) ;

- puis vient un second ensemble, plus épais (de l'ordre de $200 \mathrm{~m}$ ), formant talus, constitué de marnes noirâtres, argilo-schisteuses, tendres, appartenant au Valanginien inférieur. Ce sont ces marnes qui voient la naissance, vers la cote 1450 , du vallonnement à l'origine du talweg nord-sud ci-dessus mentionné ;

- enfin le tout est surmonté d'une nouvelle falaise (plutôt d'un ressaut) de calcaires bien lités, durs, en petits bancs, à patine sombre appartenant au Valanginien supérieur.

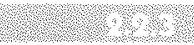

\section{Structure}

A l'échelle du site, la tectonique est extrêmement simple, les couches des quatre ensembles ci-dessus présentent un pendage vers le nord de $30^{\circ}$ à $40^{\circ}$.

\section{, s.}

\section{Les conditions météorologiques}

Les conditions météorologiques, sans être tout à fait exceptionnelles, ont contribué à augmenter dans de fortes proportions la teneur en eau des terrains superficiels. Après un mois de janvier caractérisé par du beau temps (transformation de la mince couche de neige préexistante en neige de printemps), de fortes chutes se produisent en février et mars et sont à l'origine des premières avalanches au début avril, notamment celle observée le 5 au-dessus du sanatorium. Ensuite, les 12, 13, 14 avril, des pluies abondantes alourdissent le manteau neigeux et le sol sous-jacent est certainement saturé ${ }^{2}$. La veille de la catastrophe, soit le 15 avril, survient un brusque redoux qui provoque une fusion rapide du manteau neigeux.

\footnotetext{
(2) On peut aussi supposer que des venues d'eau au travers de fractures clu substratum ont contribué à la saturation des formations superficielles, mais on n'en a aucune preuve.
} 


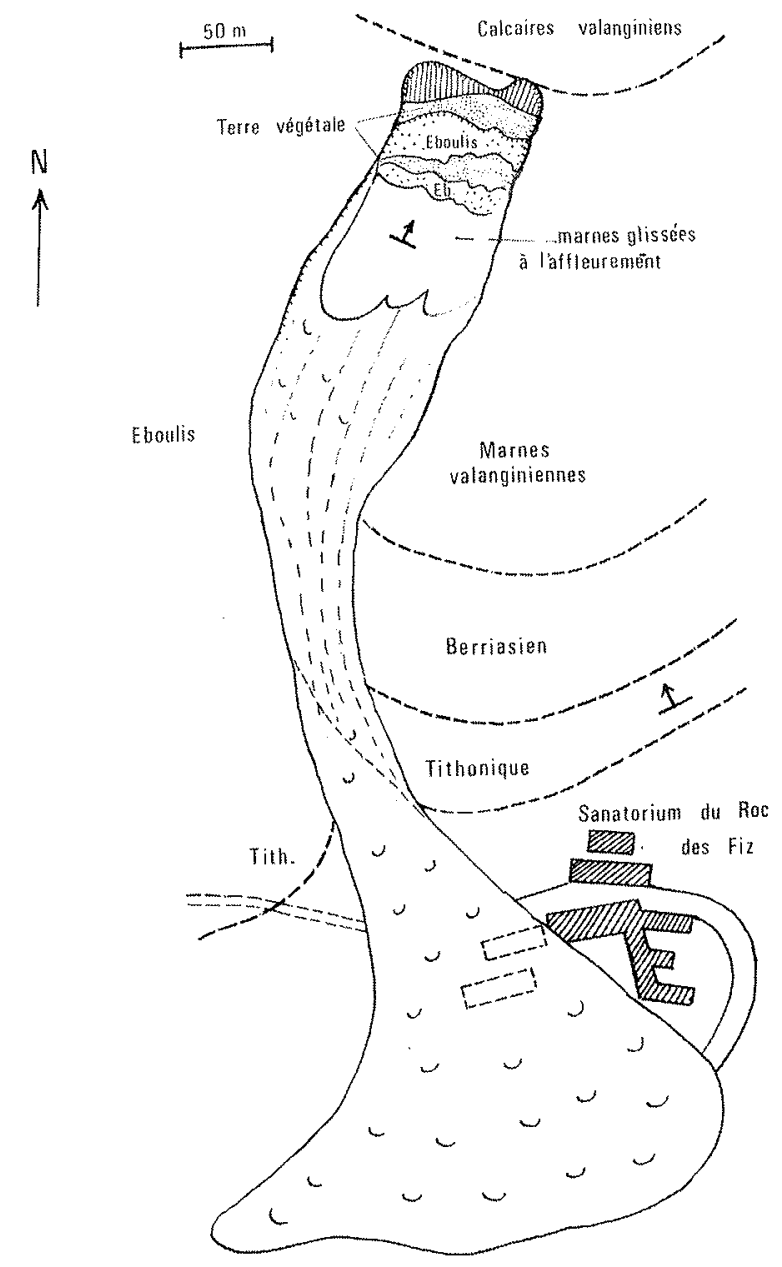

Plan de la coulée du plateau d'Assy. Map of the Plateau d'Assy flow slide.

\section{Reconstitution du mécanisme du glissement-coulée}

Le rapport de l'expert résume bien l'enchaînement des faits qui ont conduit à la catastrophe. Un glissement de formations superficielles ${ }^{3}$ (éboulis et probablement frange altérée superficielle des marnes sousjacentes) s'est produit sur le versant à $35^{\circ}$ des marnes du Valanginien inférieur. Ce glissement fut causé par la diminution des caractéristiques de résistance des marnes altérées en surface du bed-rock (conséquence de l'humidification) et par l'accroissement rapide des pressions interstitielles dans le versant. Dès la mise en mouvement et à cause des fortes teneurs en eau, la masse glissée s'est rapidement transformée en une coulée boueuse, dont le volume est évalué à plus de $50000 \mathrm{~m}^{3}$. Celle-ci a tout d'abord dévalé la pente en ligne droite depuis la cote $1550 \mathrm{~m}$ environ et, malheureusement, dès la cote 1400 , a rencontré le talweg nord-sud qui a canalisé directement la coulée sur l'aile droite du sanatorium situé à la cote 1240 environ.

\subsection{0 .92}

\section{Conclusion}

Le rappel des faits ci-dessus montre que la catastrophe résulte d'une conjonction de facteurs défavorables dont les interactions n'étaient pas imaginables à l'époque de la construction du sanatorium (1931). Si l'on pouvait à la rigueur avoir des doutes sur la stabilité des formations superficielles recouvrant les marnes du Valanginien inférieur, aucun mouvement de ce type n'avait été répertorié dans le secteur. Les conditions pluvio-nivologiques exceptionnelles ont sans conteste déclenché le mouvement, mais surtout ont complètement modifié la

(3) J. Debelmas avait initialement envisagé qu'un compartiment de marnes valanginiennes découpé par une faille avait participé au glissement. C'est l'interprétation qui est figurée sur la coupe de la figure 5. En fait un examen attentif de photographies réalisées ultérieurement a montré qu'il n'en était rien. Du reste aucun bloc de marnes n'a été retrouvé dans la masse de la coulée.

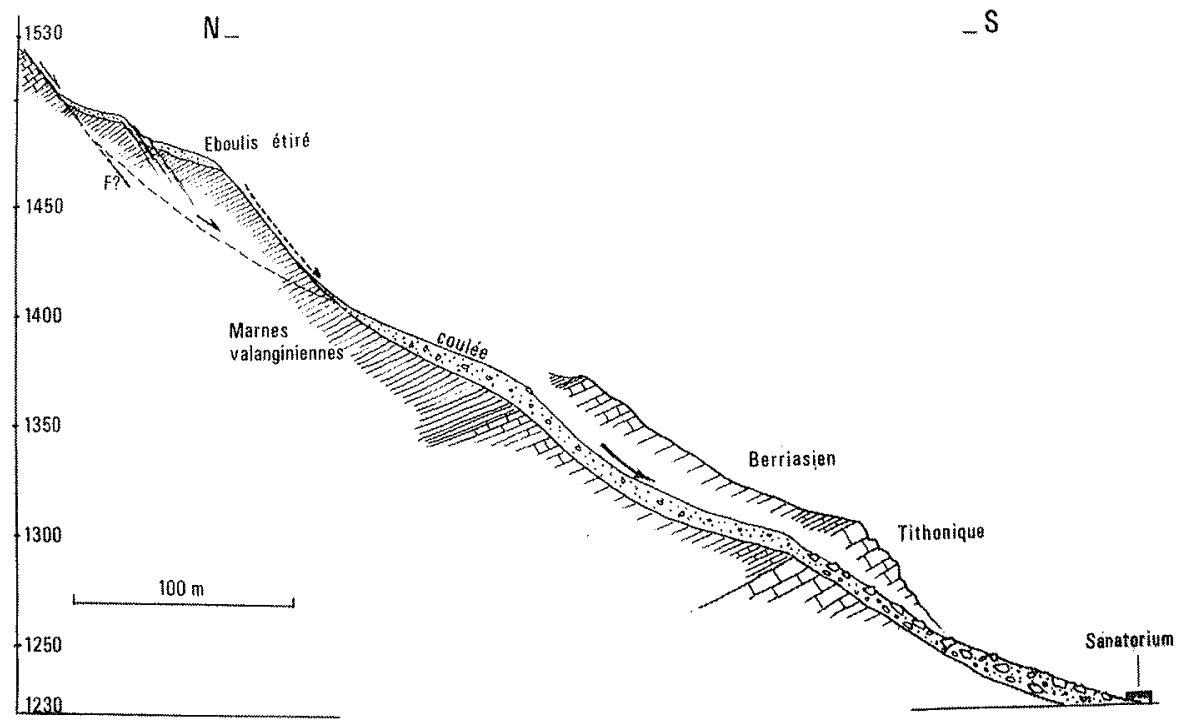

Iic. 5 Coupe de la coulée du plateau d'Assy. Section of the Plateau d'Assy flow slide. 
rhéologie de la masse en mouvement, transformée en coulée, d'où une vitesse de propagation plus grande et une extension considérable de la zone atteinte. Enfin une disposition morphologique très particulière rencontrée par la coulée (existence du petit talweg débouchant sur le sanatorium) s'est révélée meurtrière. Là encore ce fait ne pouvait être prévu : dans l'état initial, ce talweg était à peine visible car encombré d'une végétation en partie arborée. Il n'empêche que cette catastrophe a constitué un événement déclencheur dans le développement d'une politique de prévention des risques naturels.

\section{3}

\section{La catastrophe du plateau d'Assy, point de départ d'une cartographie d'aléa moderne}

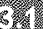

\section{Les travaux méthodologiques}

C'est au début des années 70 que la méthodologie de cartographie des phénomènes d'instabilité actifs ou potentiels a été mise au point (groupe de travail ZERMOS, cf. infra $\$ 4.2$ ). Elle repose sur une phase d'analyse (récolte des données historiques, géologiques et morphologiques; photo-interprétation; levés de terrain) et sur une phase de synthèse au cours de laquelle le géologue définit les critères qui vont lui permettre d'affecter des niveaux d'aléa aux différentes zones (LCPC-CFGI, 2000). La recherche des événements passés est essentielle aussi sur un plan pédagogique, pour éviter le classique « ... mais nous ne savions pas ! ».

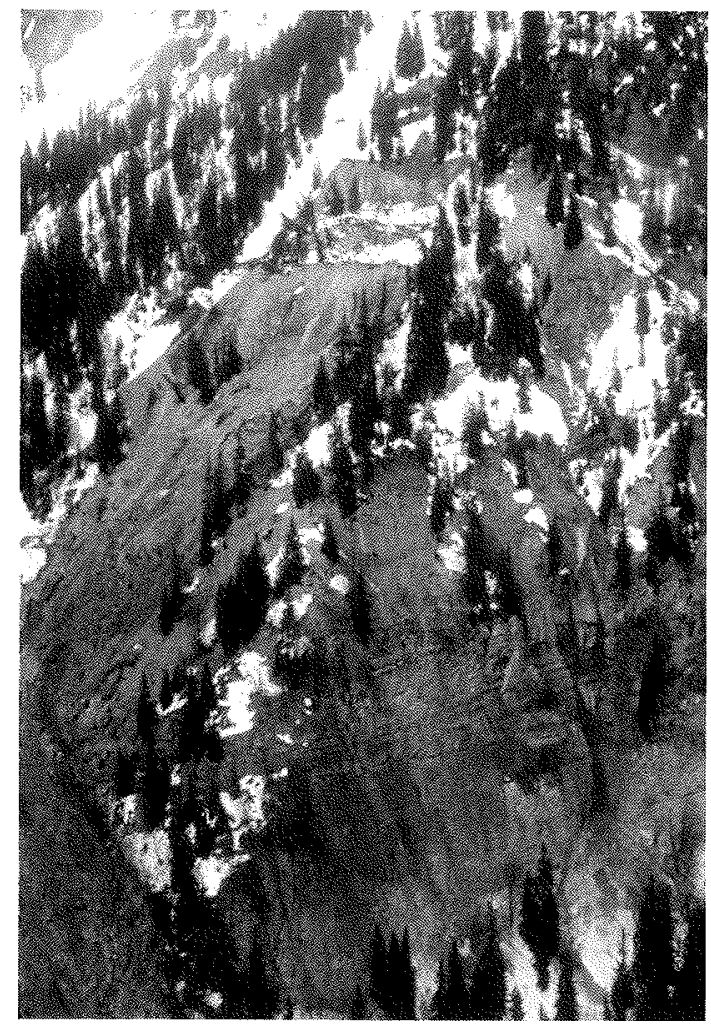

กic. 6 Vue de la zone de départ de la coulée du plateau d'Assy.

View of the upper part of the Plateau d'Assy flow slide.

\section{3.}

\section{L'adaptation des techniques cartographiques}

Pour satisfaire les demandes nouvelles, les cyéologues durent se spécialiser et adapter leurs techniques cartographiques. Cela fut bien facilité par la généralisation des ( documents techniques provisoires » de l'IGN à l'échelle 1/10 000 utilisés pour l'édition des cartes topographiques à 1/25000, ce qui fit faire un progrès considérable à la cartographie géologique des Alpes en raison de la possibilité offerte de représenter des détails qui sans cela n'auraient pas pu figurer sur la carte. Les levés de cartes dites de ( risques naturels ) ont largement bénéficié de cet avantage.

Parallèlement, la mise à disposition de photographies aériennes prises à différentes dates se développait et leur utilisation se généralisait; elles se révélaient particulièrement favorables à l'identification des terrains en mouvement.

\section{8.}

\section{La délimitation des zones affectées par les instabilités de versant}

L'exemple de la catastrophe du plateau d'Assy a montré quelle pouvait être l'importance de la prévision de l'extension des surfaces susceptibles d'être affectées par un mouvement de versant. A cet égard, deux cas peuvent se présenter :

- les phénomènes, quelle que soit leur nature, où les déplacements des matériaux sont limités. Les dommages restent à peu près circonscrits à la zone active, les vitesses de déformation étant généralement lentes. Le risque pour les personnes est très faible, mais l'utilisation des terrains, notamment pour la construction, doit être réglementée ;

- les phénomènes, amorcés sur une surface donnée, mais tels que la masse en mouvement se désolidarise de son point de départ pour atteindre des zones éloignées, comme ce fut le cas au plateau d'Assy. Il s'agit, d'une part, des chutes de blocs et éboulements rocheux, d'autre part, des laves torrentielles et coulées diverses.

Sur un versant de montagne, l'énergie potentielle disponible, entre l'altitude de départ et le fond de la vallée, peut être considérable. Trois cas peuvent typiquement se rencontrer :

- celui d'une masse dans laquelle une bonne part de l'énergie étant dissipée par la déformation interne à la masse et le frottement basal, l'énergie cinétique résultante restera faible et la distance parcourue limitée. Ce premier cas correspond, entre autres, aux innombrables glissements post-glaciaires qui ont affecté les vallées alpines, généralement qualifiés de glissements anciens. C'est aussi le cas du glissement de La Clapière (Alpes-Maritimes), qui mobilise plus de $50 \mathrm{hm}^{3}$ de te rain mais dont l'énergie cinétique au maximum de sa vitesse était inférieure à 1 Joule (Durville, 1992);

- celui d'une masse rocheuse cohérente, de comportement fragile, pour laquelle la fragmentation en cours de mouvernent sera importante et les frottements faibles (éléments à trajectoires en partie aériennes), en ne dissipant que peu d'énergie : la plus grande partie de l'énergie potentielle se transformera alors en énergie 
cinétique, ce qui signifie grande vitesse et déplacement important. Il s'agit notamment des phénomènes qualifiés d'avalanches rocheuses;

- celui d'une masse de sol en glissement qui, du fait de teneurs en eau très élevées, se transforme en un fluide visqueux, comme au plateau d'Assy. Son mouvement relève alors d'une rhéologie particulière et la prévision de la zone d'atteinte est très délicate. On peut envisager, avec prudence, une modélisation.

Cette question de la distance parcourue se révèle donc très difficile puisqu'il faut prévoir, de façon qualitative et quantitative, le comportement mécanique de la masse en mouvement et les modifications de ce comportement au cours du mouvement. Dans les zonages cartographiques qui ont été réalisés ces dernières décennies, la détermination de la zone d'extension aval des phénomènes reste une source d'erreur ou de discussion importante.

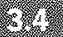

\section{La prévision temporelle}

La menace d'une rupture dans un versant, lorsqu'elle est identifiée, et en fonction des conséquences possibles pour les personnes et les biens, peut conduire à mettre en place un système d'alerte et à prendre des décisions administratives telles qu'une limitation d'usage ou une évacuation. De telles mesures nécessitent l'établissement d'une prévision précise de l'évolution du phénomène.

Les méthodes actuellement utilisées pour ce faire reposent sur le suivi des déplacements dans le temps et sur une extrapolation de ceux-ci. Ceci impose une instrumentation et des mesures dont le volume et le coût varient beaucoup en fonction de la surface à couvrir et, comme dans tout cas de mesure physique, le stade final est une phase d'interprétation. Les exemples connus connaissent à peu près autant de succès que d'échecs. Si l'on fait une prévision précise, et qu'elle soit cémentie par les faits, cela a généralement pour conséquence un refus d'accorder foi à la méthode pour le futur. La législation et la réglementation ont de la peine à s'adapter à une telle situation. En fait, dans la plupart des cas, la prévision devrait adopter une formulation probabiliste, laquelle n'est guère appréciée des juristes. Pourtant, en matière de risque naturel hydrologique, les prévisions de débits des crues sont essentiellement probabilistes, et cela est admis. Une différence majeure sépare toutefois ces deux cas : les crues, en un point donné des cours d'eau, sont répétitives, à l'opposé des glissements de terrain.

Dans le cas du plateau d'Assy, pour répondre à la question du préfet quant à la prévision, il aurait fallu déceler l'amorce d'un glissement dans un sol soumis à des variations de pressions interstitielles, en un endroit précis du versant. Il aurait fallu ensuite mettre en place des piézomètres, dont on aurait été sûr qu'ils fonctionnent bien pendant un long laps de temps, et effectuer des mesures topographiques. La question de la prévision temporelle, dont personne à l'époque ne concevait l'arrière-plan scientifique et technique, était donc réglée dans ce cas (et il en serait de même actuellement) : cette prévision était parfaitement impossible. C'est pourquoi la cartographie d'aléa constitue - encore aujourd'hui - le socle de toute prévention, même si le perfectionnement des techniques (interférométrie radar, par exemple) permettra de plus en plus de détecter assez tôt des mouvements précurseurs.

\section{La réponse de la puissance publique}

II faut tout d'abord rappeler que, peu de temps avant la catastrophe d'Assy, une avalanche meurtrière avait balayé un chalet à Val-d'Isère, causant 39 victimes. L'attention du public et, par conséquent, celle de la puissance publique, se trouvait donc brutalement focalisée sur les risques naturels en montagne pour constater que les textes de référence étaient très peu nombreux et imprécis et que l'administration était bien en peine d'exercer l'obligation d'agir que lui impose le droit français. La catastrophe du plateau d'Assy mit cruellement en lumière les principales lacunes qu'il convenait de combler au plus vite, relatives:

- à l'information, tant du public que des autorités, sur les risques. Il s'agit en fait d'attirer l'attention sur tout phénomène naturel susceptible d'avoir des conséquences graves pour les personnes ou pour les biens ;

- à l'établissement d'une législation et d'une réglementation spécifique.

\section{7)}

\section{Les réactions immédiates}

Lors de la séance du 29 avril 1970 à l'Assemblée nationale, dans le cadre des questions d'actualité, le député J. Morellon déclarait : “ Il s’agit de savoir si cette tragédie est la conséquence d'une fatalité ou si elle était scientifiquement prévisible. [...] Il est certain qu'inéluctablement se produiront d'autres mouve. ments de ce genre dans les années peut-être, dans les siècles sûrement, à venir. Ne faudrait-il pas prévoir et réglementer la construction en zone montagneuse en s'entourant du maximum de garanties de sécurité ? m.

La mission interministérielle d'étude sur la sécurité des stations de montagne, présidée par J. Saunier, inspecteur général de l'administration, mission créée après l'avalanche de Val-d'Isère mais dont le champ est élargi après l'événement d'Assy, produit son rapport en juillet 1970. Bien qu'essentiellement consacré aux avalanches, ce rapport formule plusieurs recommandations applicables aux risques de mouvements de terrain ; en particulier, il propose de réaliser des plans des « zones exposées aux risques naturels, établis par commune » avec une " délimitation précise des zones exposées et des indications sur l'intensité des phénomènes redoutés m, plans qui devraient être utilisés pour l'application du décret de 1961.

Plusieurs circulaires ont suivi, concernant la prévention du risque d'avalanche ou de mouvement de terrain ; en particulier, celle du ministre de l'Agriculture, en date du $1^{\text {er }}$ février 1971, demande que soient confectionnés, en application du rapport Saunier, des ( plans des zones exposées aux risques naturels ). Le décret du 28 octobre 1970 relatif aux Plans d'occupation des sols (POS) stipule que ceux-ci doivent prendre en compte les zones où " l'existence de risques naturels tels que inondation, érosion, affaissements, éboulements, avalanches, justifie que soient interdites ou soumises à des conditions spéciales les constructions ou installations de toute nature $)$.

Par ailleurs, si le Tribunal administratif de Grenoble a reconnu en 1974 (arrêt confirmé par le Conseil d'État en 1986) la responsabilité conjointe de la commune et 
de l'État dans l'accident de 1970 à Val-d'Isère, du fait du manque d'études approfondies des zones exposées et du retard mis à délimiter ces zones, il n'y a rien eu de tel dans le cas du sanatorium du Roc des Fiz; il faut dire que des avalanches importantes avaient eu lieu dans le passé sur le site de Val-d'Isère, alors qu'aucun glissement n'était connu dans le versant dominant le sanatorium. Des actions en responsabilité civile ont cependant visé la commune de Passy, propriétaire des terrains d'où la coulée est partie. Le Tribunal de Grande Instance de Bonneville a reconnu en 1978 le caractère de force majeure, exonérant ainsi la commune ; en revanche, la cour d'appel de Chambéry, en 1980, a déclaré la commune " responsable, en sa qualité de gardienne, des conséquences du glissement de terrain »), mais pour moitié seulement. Le raisonnement de la cour d'appel est assez tortueux : il s'appuie sur le rapport de l'expert pour établir que le (c vice du terrain », à savoir l'altération de la couche superficielle susceptible de se gorger d'eau, n'était pas quelque chose d'inimaginable (d'où la part de responsabilité), mais que les circonstances météorologiques exceptionnelles relevaient de la force majeure (d'où l'exonération pour moitié).

\section{$2+2$}

\section{Les développements ultérieurs}

Plusieurs actions se mettent en place dans les années 70.

En 1974, le BRGM ${ }^{4}$ et le $\mathrm{LCPC}^{5}$ réalisent conjointement une enquête préliminaire sur l'existence des ( zones exposées à des risques liés aux mouvements du sol et du sous-sol » (ZERMOS). Ils s'attachent à évaluer, dans chaque département, la surface concernée par les mouvements de terrain et l'opportunité d'une cartographie de type ZERMOS.

Le Plan ZERMOS est financé par des crédits alloués à la direction de la Sécurité civile au ministère de l'Intérieur; la réalisation en est confiée au BRGM qui s'adjoint la collaboration des laboratoires des Ponts et Chaussées et de certains laboratoires de géologie de l'Université. Il s'agit de mettre au point une méthodologie et de réaliser plusieurs cartes de « zones exposées aux risques de mouvements du sol et du sous-sol m, préfigurant une cartographie plus systématique.

Le groupe de travail ZERMOS, présidé par Jean Goguel, a ainsi défriché le terrain, à une époque où par exemple les concepts d'aléa et de risque n'étaient pas encore clairement définis dans le domaine des instabilités de versants. Vingt-sept cartes ont été réalisées entre 1972 el 1980, à l'échelle 1/25 000 en général (Champetier de Ribes, 1987).

L'une de ces cartes, éditée par le BRGM en 1976 à l'échelle 1/20 000, concerne le territoire de Passy-Servoz (Fig. 7) ; le tracé de la coulée de 1970 est bien évidemment indiqué. Les matériaux glissés en 1970 ne sont plus en place, mais il est envisageable, compte tenu de la nature du glissement, du remaniement des matériaux glissés, de la faible protection par un couvert forestier (quarante ans après, la trace est encore bien visible sur le versant), que de nouvelles instabilités se produisent dans un futur proche, par régression amont ou remobilisation.

\footnotetext{
(4) Bureau de recherches géologiques et minières.

(5) Laboratoire central des Ponts et Chaussées.
}

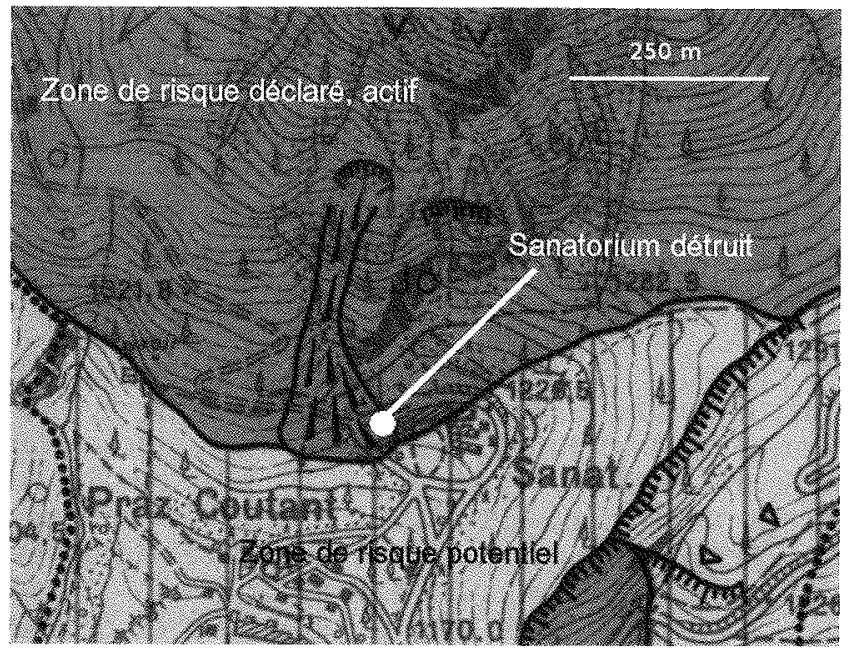

Fic. 7 Extrait de la carte ZERMOS de PassyServoz (d'après BRGM, auteur A. Pachoud, 1976).

Extract from the hazard map of Passy-Servoz.

En dépit de la qualité du travail réalisé, la carte ZERMOS de Passy-Servoz met en évidence certaines difficultés inhérentes à ce type de document - aujourd'hui quasi abandonné - compte tenu de l'échelle adoptée et des objectifs poursuivis. Tout le versant dominant le sanatorium est figuré en gris foncé (crisque déclaré, actif $)$ ), qu'il s'agisse des falaises rocheuses, des talus d'éboulis qu'elles engendrent ou des pentes boisées établies sur de la moraine ou des éboulis. La valeur des pentes ne semble pas avoir été prise en considération et la zone grise rassemble des contextes morphologiques et géologiques très différents.

Concernant l'extension de la zone gris foncé, on peut se poser la question de la justification de sa limite inférieure. N'a-t-elle pas été influencée par la coulée de 1970 ? En l'absence de celle-ci, la logique morphologique du versant n'aurait-elle pas conduit à placer la limite plus près de la falaise, et donc à exclure le sanatorium de la zone rouge foncé ? En dehors du débouché du léger talweg qu’a emprunté la coulée, le risque de propagation est-il équivalent?

Une dernière remarque concerne les hachures verticales, qui recouvrent en particulier le secteur du sanatorium. Elles traduisent un risque qualifié de ( séculaire » par l'auteur (éventualité d'un grand écroulement), et donc une zone de risque majeur, mais à long terme. L'appréciation du danger est subjective et peut certainement être discutée, en l'absence de faits chronologiquement avérés. La traduction concrète en termes de contraintes d'aménagement n'est pas facile à déterminer.

Parallèlement au plan ZERMOS, des cartes des risques liés aux mouvements de terrain (CRAM) sont dressées dans les Alpes-Maritimes par le laboratoire des Ponts et Chaussées de Nice.

Une circulaire du ministère de l'Intérieur (26 novembre 1974) instaure une liste départementale de géotechniciens agréés en malière de mouvements du sol et du sous-sol. Toutefois cette circulaire sera très inégalement applicuée et aura peu de suites réellement opérationnelles 
5

\section{Les évolutions plus récentes}

Sous l'impulsion d'Haroun Tazieff, la loi du 13 juillet 1982 relative à l'indemnisation des catastrophes naturelles est promulguée, qui crée les Plans d'exposition aux risques (PER), remplacés en 1995 par les Plans de prévention des risques naturels prévisibles (PPR). Il est bien connu que de nombreuses catastrophes, principalement crues et inondations plus ou moins brutales, ont conduit ces dernières années à un fort développement de lois et règlements relatifs à la prévention des risques naturels.

Un cas particulier mérite d'être signalé, c'est l'expropriation pour risques naturels majeurs, instituée par la loi du 2 février 1995 et qui trouve son origine dans des situations de péril non imminent mais considéré comme certain. Dans son rapport sur le projet de loi, le sénateur J.-F. Legrand évocue deux cas précis: " le hameau de l'Île-Falcon au pied de la Séchilienne, menacé par l'effondrement prochain et inévitable de plusieurs dizaines de millions de $\mathrm{m}^{3}$ de terre m, ainsi que des habitations situées au-dessus de carrières de gypse abandonnées, sur la butte de l'Hautil en région parisienne. L'objet de l'expropriation pour risques naturels majeurs est, selon la circulaire interministérielle du 10 juillet 1996, de donner à l’État « la possibilité de faire évacuer, dans des conditions justes et équitables, les personnes habitant dans les zones soumises à des risques importants prévisibles ». A Séchilienne par exemple, le décret d'expropriation, en date du 31 mai 1997 , visait le lotissement de l'île-Falcon, une petite centrale EdF et une papeterie, c'est-à-dire des biens concernés par l'éboulement lui-même ou par ses conséquences hydrologiques (rupture par débordement du barrage formé par les éboulis, et inondation à l'aval).
En fait, un autre cas avait déjà mis en évidence une lacune dans le dispositif mis en place par la loi de 1982 précitée. A Saint-Étienne-de-Tinée, le grand glissement de La Clapière était surveillé depuis 1976 ; le mouvement lent (quelques centimètres par jour) engendrait des chutes de blocs issus d'une corniche rocheuse située à mi-pente, la barre d'Iglière. Une scierie (Fig. 8) se trouvait dans un secteur directement soumis au risque de chutes de blocs et un arrêté municipal d'interdiction d'accès, pris en 1988, avait inclus dans son périmètre le bâtiment de la scierie ${ }^{6}$. L'idée d'une « indemnisation avant réalisation du dommage » avait alors été avancée. A titre exceptionnel, un accord avait pu être trouvé et le transfert de l'établissement a été effectué aux frais de la compagnie d'assurance, devançant ainsi le dommage, mais il était clair que, pour apporter une réponse satisfaisante à ce type de situation, un texte spécifique était nécessaire.

Comme on le voit, non seulement l'occurrence d'une catastrophe, mais aussi la seule menace d'un mouvement de terrain, peuvent servir de catalyseurs pour l'établissement d'une nouvelle législation...

Avec le recul, il apparaît que les deux catastrophes de 1970 constituent des événements charnières dans la politicue de prévention des instabilités en montagne et, plus largement, des risques naturels dans notre pays, même si par ailleurs on peut invoquer une évolution plus progressive, celle des mentalités qui passent en quelques décennies d'une acceptation résignée de la fatalité à la quasi-nécessité de trouver des entités responsables, susceptibles en particulier d'indemniser les victimes.

(6) Le PPR approuvé en 1997 placera cette scierie en zone “ rouge » inconstructible, à la fois pour les chutes de blocs et pour la menace du glissement de grande ampleur.

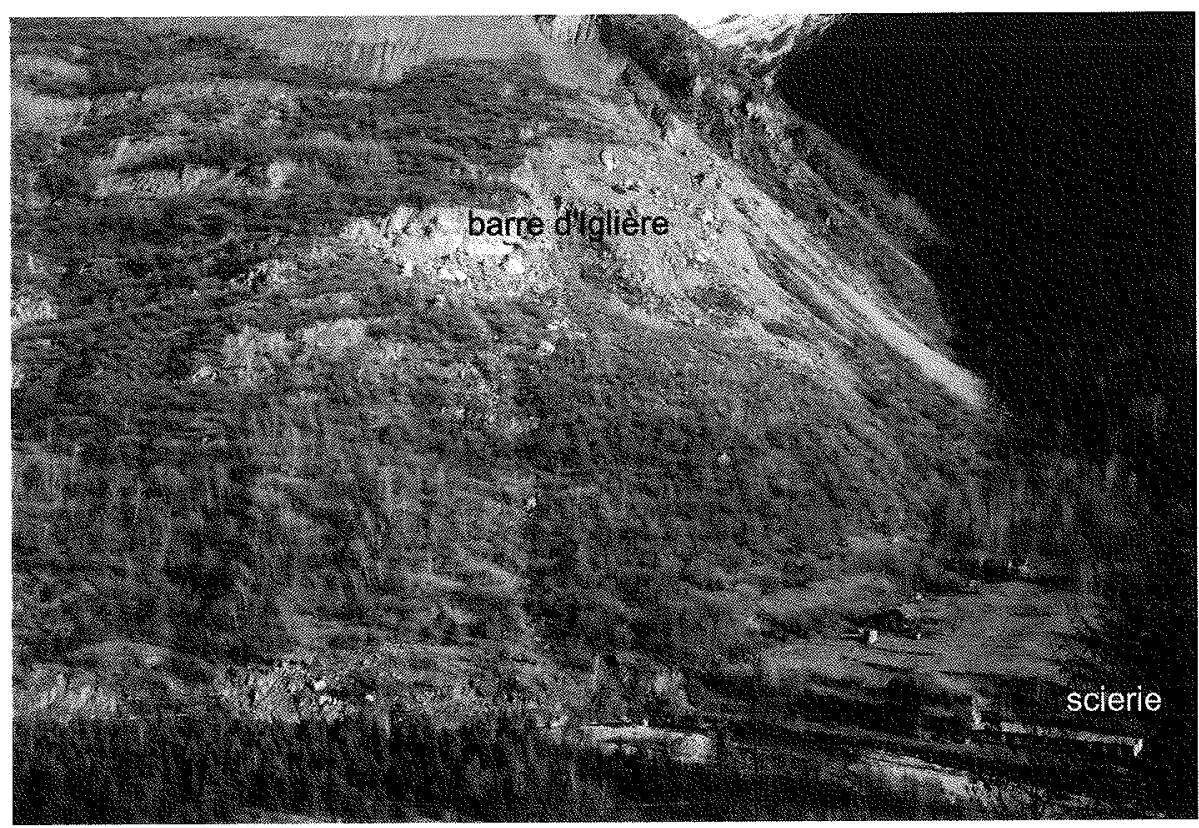

FIG 8 Glissement de La Clapière (Alpes-Maritimes) : la partie sud-est du glissement avec la barre rocheuse d'Iglière et la scierie évacuée. Picture of La Clapière (Alpes-Maritimes, France) landslide, showing the Iglière cliff and the evacuated sawmill. 


\section{Bibliographie}

Antoine P., Biarez J., Desvarreux P., Mougin J.-P. - Les problèmes posés par la stabilité des pentes dans les régions montagneuses. Géologie alpine, t. 47, 1971 , p. $5-24$

Antoine P., Pachoud A. - Enseignements tirés de deux essais de cartographie systématique de glissements de terrain Bull. Liaison Labo. P. et Ch., 150-151, 1976, p. 31-39.

Antoine P. - Some unexpected features of landslides. Proceedings 5th International Symposium on Landslides, C. Bonnard ed., Lausanne, Balkema, 1988, p. 13891392.
Besson L.. - Les risques naturels : de la connaissance pratique à la gestion administrative. Techni-Cités, 2005, 592 p.

Champetier de Ribes G. - La cartographie des mouvements de terrain. Des ZERMOS aux PER. Bull. Liaison Labo. P. et Ch., 150-151, 1987, p. 9-19.

Debelmas J. - Les géologues alpins et les Alpes : 150 ans d'efforts et de clécouvertes. Géologues, $n^{\circ} 160,2009$, p. 16-19.

Durville J.-L. - Mécanismes et modèles de comportement des grands mouvements de versants. Bull. A.I.G.I., n ${ }^{\circ} 45,1992$, p. 25-42.
Froment L. - Le Claps et l'ancien lac de Lucen-Diois, CGP Antibes, 1983, 42 p., $2^{\circ}$ éd.

Kerckhove C. - Feuille Barcelonnette de la carte géologique de la France à 1/50 000 et notice explicative, BRGM Orléans, 1971.

LCPC-CFGI - Caractérisation et cartographie de l'aléa dû aux mouvements de terrain. Collection Environnement. LCPC, $2000,90 \mathrm{p}$.

Liochon M. - Aspects juridicues des problèmes de glissements de terrain. Bull. Liaison Labo. P. et Ch., spécial, mars 1976 , p. $13-20$

Pachoud A. - Notre-Dame de Myans. Trésors de la Savoie, 1983, 127 p. 